\title{
Evaluation of All the Material Constants of PMN-28\%PT Piezoelectric Single Crystals for Acoustic Transducers
}

\author{
Jinwook Kim ${ }^{1}$, Cheeyoung Joh ${ }^{2}$ and Yongrae Roh ${ }^{1, *}$ \\ 'School of Mechanical Engineering, Kyungpook National University, Daegu 702-701, Korea \\ ${ }^{2}$ Agency for Defense Development, Changwon 645-600, Korea
}

(Received October 22, 2012; accepted April 8, 2013)

Key words: material constants, PMN-PT, single crystals, finite element analysis, resonator measurement

In this study, all the elastic, piezoelectric, and dielectric constants of lead magnesium niobate-28\%lead titanate (PMN-28\%PT) single crystals have been determined by the resonance method. In comparison with other PMN-PT crystal compositions characterized so far, PMN-28\% PT crystals offer better structural reliability and easy growth controllability. The PMN-28\%PT single crystals were grown by the Bridgman method to have a tetragonal symmetry of $4 \mathrm{~mm}$, and thus have eleven independent material constants: six elastic constants, two dielectric constants and three piezoelectric constants. The eleven constants were determined through measurement and analysis of only the impedance spectra of six different types of PMN-PT resonators: length extensional (LE), thickness extensional (TE), thickness shear (TS)-mode, and length thickness extensional (LTE) resonators, and two variations of the LTE resonators, $X Y t 45^{\circ}$ and $Z X t 45^{\circ}$. The characterization method in this work yields all the material constants of PMN-28\%PT crystals through consistent and systematic resonance measurements. The validity of the characterization method and accuracy of the determined material constants are demonstrated through comparison of the experimental impedance spectra with numerically obtained impedance spectra of the resonators having the derived constants.

\section{Introduction}

The piezoelectric single-crystal $\mathrm{Pb}\left(\mathrm{Mg}_{1 / 3} \mathrm{Nb}_{2 / 3}\right) \mathrm{O}_{3}-\mathrm{PbTiO}_{3}$ (PMN-PT) has attracted much interest from the acoustic transducer community for benefits such as increased bandwidth, sensitivity and source levels due to the high electromechanical coupling factor and piezoelectric constants of the crystal. ${ }^{(1-4)}$ The crystals also have disadvantages such as a low phase-transition temperature, high cost, and limitation of available sizes. Thus, the use of the crystals requires more careful modeling and design processes than

${ }^{*}$ Corresponding author: e-mail: yryong@knu.ac.kr 
in the case of dealing with conventional ceramics. Models to analyze the performance of acoustic transducers would be useful engineering tools that could facilitate the design. However, the models are only as effective as the material properties used in them. Hence, in this work, all the elastic, dielectric, and piezoelectric material constants of the PMN-28\% PT single crystal have been measured by the resonance method, and their accuracy has been confirmed through numerical analysis of the electromechanical properties of crystal samples.

Several different methods are available for estimating the material constants of piezoelectric crystals, such as the ultrasonic pulse-echo method, resonator measurement and the use of specific instruments to measure particular constants. ${ }^{(5-10)}$ Moreover, several iterative methods have been proposed for evaluating complex material constants by deriving electrical impedance equations for crystals of different geometries. ${ }^{(11-14)}$ Kwok et al. evaluated the performance of various characterization methods and compared the advantages and disadvantages among the methods. ${ }^{(14)}$ In terms of measurement accuracy, Matko demonstrated that the switching methods for crystal sensing devices outperforms conventional measurement methods because the switching method highly improves second-to-second and minute-to-minute frequency stability. ${ }^{(15,16)}$ Among the characterization methods, the resonance method is regarded as providing reasonably accurate results and being easy to use as long as crystal samples of various shapes are available. Using the resonance method in combination with other techniques, Zhang et al. evaluated the orientation dependence and material properties of PMN-33\%PT single crystals, ${ }^{(8)}$ and Lee and Roh characterized PMN-32\%PT single crystals grown by the solid-state crystal growth method. ${ }^{(17)}$ However, in most of the reported works, the resonance method has been used to evaluate particular constants of the crystal, not the whole material constant matrices.

There are also several different methods of growing PMN-PT crystals: the flux method (using $\mathrm{PbO}$ flux), Bridgman method, solution Bridgman method (using $\mathrm{PbO}$ flux), and solid-state crystal growth. ${ }^{(18)}$ Furthermore, PMN-PT crystals have excellent piezoelectric properties only near the morphotropic phase boundary where the solid solution changes its structure during cooling from rhombohedral to tetragonal, depending on its composition. ${ }^{(19,20)}$ Hence, crystal properties are strongly dependent on the growth method and composition. ${ }^{(21)}$

In this study, PMN-PT single crystals grown by the Bridgman method to have a composition of PMN-28\% PT are characterized by the resonance method. In comparison with other PMN-PT crystal compositions characterized so far, PMN-28\%PT crystals offer better structural reliability and easy growth controllability. ${ }^{(21,22)}$ The PMN-28\%PT single crystals belong to the tetragonal symmetry group of $4 \mathrm{~mm}$, and thus have eleven independent material constants: six elastic constants, two dielectric constants, and three piezoelectric constants. ${ }^{(23)}$ All eleven constants are determined through measurement and analysis of only the electromechanical impedance spectra of six different types of PMN-28\%PT resonators. The method developed in this work can yield all the material constants of PMN-28\% PT crystals through consistent and systematic resonance measurements. To verify the accuracy of the determined material constants and the validity of the characterization method, the experimental impedance spectra are compared and discussed with numerically obtained impedance spectra of the resonators having the derived constants. 


\section{Material Constants of PMN-28\%PT Crystals}

The PMN-28\%PT crystals belong to the tetragonal $4 \mathrm{~mm}$ symmetry group. The crystals have six independent elastic constants c, two independent dielectric constants $\varepsilon$, and three independent piezoelectric stress constants e, giving a total of eleven independent constants, as presented in eqs. (1)-(3). ${ }^{(23)}$ The superscripts $\mathrm{E}$ and $\mathrm{S}$ indicate that the constants were measured at a constant electric field $\mathrm{E}$ and constant strain $\mathrm{S}$, respectively.

$$
\begin{gathered}
c^{\mathrm{E}}=\left[\begin{array}{cccccc}
c_{11}^{\mathrm{E}} & c_{12}^{\mathrm{E}} & c_{13}^{\mathrm{E}} & 0 & 0 & 0 \\
c_{12}^{\mathrm{E}} & c_{11}^{\mathrm{E}} & c_{13}^{\mathrm{E}} & 0 & 0 & 0 \\
c_{13}^{\mathrm{E}} & c_{13}^{\mathrm{E}} & c_{33}^{\mathrm{E}} & 0 & 0 & 0 \\
0 & 0 & 0 & c_{44}^{\mathrm{E}} & 0 & 0 \\
0 & 0 & 0 & 0 & c_{44}^{\mathrm{E}} & 0 \\
0 & 0 & 0 & 0 & 0 & c_{66}^{\mathrm{E}}
\end{array}\right]\left[\mathrm{N} / \mathrm{m}^{2}\right] \\
e=\left[\begin{array}{cccccc}
0 & 0 & 0 & 0 & e_{15} & 0 \\
0 & 0 & 0 & e_{15} & 0 & 0 \\
e_{31} & e_{31} & e_{33} & 0 & 0 & 0
\end{array}\right]\left[\mathrm{c} / \mathrm{m}^{2}\right] \\
\varepsilon^{\mathrm{s}}=\left[\begin{array}{cccc}
\varepsilon_{11}^{\mathrm{S}} & 0 & 0 \\
0 & \varepsilon_{11}^{\mathrm{s}} & 0 \\
0 & 0 & \varepsilon_{33}^{\mathrm{s}}
\end{array}\right][\mathrm{F} / \mathrm{m}]
\end{gathered}
$$

\section{Resonator Measurement}

The electromechanical properties of piezoelectric crystals are determined as a function of the elastic, dielectric and piezoelectric constants of the crystals. In other words, careful analysis of the electromechanical features can reveal the material constants of the crystals. The resonance measurement technique involves observing the resonant and antiresonant frequencies of crystal samples of various geometries to derive the material constants directly related to the particular mode of vibration of each sample. ${ }^{(10,24,25)}$ For the generation of clear resonance modes without mode coupling, the crystal resonators must have specific geometries suitable for particular modes of vibration. To identify the eleven independent material constants of the PMN28\%PT single crystals, six different resonators have been analyzed in this work: length extensional (LE), thickness extensional (TE), thickness shear (TS), and length thickness extensional (LTE) resonators, and two variations of the LTE resonators, $X Y t 45^{\circ}$ and $Z X t 45^{\circ} .{ }^{(25,26)}$ Each resonator was thus named to represent its principal resonant mode, adopting the definition and nomenclature given in the IEEE Standard on Piezoelectricity. ${ }^{(25,26)}$ In the notation for the $X Y t 45^{\circ}$ resonator, the first character $X$ means that the direction of the resonator thickness is along the crystal $X$ axis, the second character $Y$ means that the direction of the resonator length is along the crystal $Y$ axis, and $t 45^{\circ}$ means that the resonator is rotated around its thickness direction $t$ by $45^{\circ}{ }^{(25,26)}$ The same notations are also applied to the $Z X t 45^{\circ}$ model. The specific geometry and properties of each resonator are described below and in Figs. 1 to 6. 


\subsection{Thickness extensional (TE)-mode resonator}

The TE mode occurs in a piezoelectric crystal plate with a width much larger than its thickness, as shown in Fig. 1. The symbols $l, w$, and $t$ indicate the length, width, and thickness of the resonators, respectively. For clear observation of the TE mode, the dimensions of the plate should satisfy the conditions $w>10 t$ and $l>10 t .^{(24,25)}$ The figure shows both the initial shape (dotted line) and deformed shape of the resonator. The thick arrow inside the plate denotes the polarization direction of the resonator. The resonator with this geometry has little fringing effect across its boundaries and thus is used to measure the quasi-static capacitance $C_{3}$ of the crystal, which leads to the dielectric permittivity along the thickness direction $\varepsilon_{33}^{\mathrm{T}}$ via eq. (4). $A$ is the cross-sectional area of the plate and $\varepsilon_{0}$ is the permittivity of the free space, which is $8.854 \times 10^{-12} \mathrm{C} / \mathrm{m}$.

Below, $f_{\mathrm{r}}$ and $f_{\mathrm{a}}$ refer to the resonant frequency and antiresonant frequency, respectively, of the TE-mode vibration. With these $f_{\mathrm{r}}$ and $f_{\mathrm{a}}, c_{33}^{\mathrm{E}}$ is derived from eqs. (5)-(7), where $k_{\mathrm{t}}$ denotes the coupling coefficient of the TE-mode vibration. ${ }^{(10,24,27)}$ The superscripts T, $\mathrm{D}$, and $\mathrm{E}$ indicate that the material constants were measured at a constant stress, electric displacement field, and electric field, respectively. ${ }^{(23)}$

$$
\begin{gathered}
\varepsilon_{33}^{\mathrm{T}}=C_{3} \cdot \frac{t}{A} \\
k_{t}^{2}=\frac{\pi}{2} \frac{f_{\mathrm{r}}}{f_{\mathrm{a}}} \tan \left(\frac{\pi}{2} \frac{f_{\mathrm{a}}-f_{\mathrm{r}}}{f_{\mathrm{a}}}\right) \\
c_{33}^{\mathrm{D}}=\rho\left(2 t f_{\mathrm{a}}\right)^{2} \\
c_{33}^{\mathrm{E}}=c_{33}^{\mathrm{D}}\left(1-k_{\mathrm{t}}^{2}\right)
\end{gathered}
$$

\subsection{Length extensional (LE)-mode resonator}

The LE resonator model is a thin rod with the specific geometric conditions of $l>$ $5 w, l>5 t$. As illustrated in Fig. 2, this resonator vibrates along the length direction. From the values of $f_{\mathrm{r}}$ and $f_{\mathrm{a}}$ for this LE-mode vibration, the electromechanical coupling
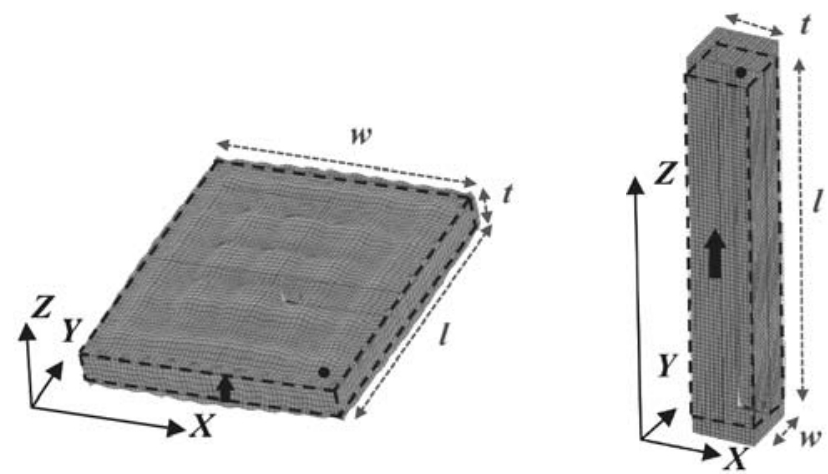

Fig. 1 (left). TE-mode resonator.

Fig. 2 (right). LE-mode resonator. 
coefficient $k_{33}$, elastic compliances $s_{33}^{\mathrm{D}}$ and $s_{33}^{\mathrm{E}}$, and piezoelectric strain constant $d_{33}$ are respectively derived from eqs. (8)-(11). ${ }^{(10,24,27)}$

$$
\begin{gathered}
k_{33}^{2}=\frac{\pi}{2} \frac{f_{\mathrm{r}}}{f_{\mathrm{a}}} \tan \left(\frac{\pi}{2} \frac{f_{\mathrm{a}}-f_{\mathrm{r}}}{f_{\mathrm{a}}}\right) \\
s_{33}^{\mathrm{D}}=\frac{1}{\rho\left(2 l f_{\mathrm{a}}\right)^{2}} \\
s_{33}^{\mathrm{E}}=\frac{s_{33}^{\mathrm{D}}}{1-k_{33}^{2}} \\
d_{33}=k_{33} \sqrt{s_{33}^{\mathrm{E}} \cdot \varepsilon_{33}^{\mathrm{T}}}
\end{gathered}
$$

\subsection{Thickness shear (TS)-mode resonator}

The thickness shear resonance of a thin plate is investigated to identify the material constants related to the shear vibration of the crystal. For this mode of vibration, the resonator should satisfy the dimensional requirements of $w>10 t$ and $l>10 t$. For the resonator model in Fig. 3, the electric field is applied along the thickness direction, which is perpendicular to the polarization direction. With this TS model, the elastic stiffnesses $c_{44}^{\mathrm{D}}$ and $c_{44}^{\mathrm{E}}$, elastic compliance $s_{44}^{\mathrm{E}}$, piezoelectric constant $d_{15}$, and dielectric permittivity are derived from eqs. (12)-(17), where $k_{15}$ is the coupling coefficient of the TS mode of vibration. ${ }^{(10,24,27)} C_{1}$ is the quasi-static capacitance across the thickness and A is the crosssectional area of the TS resonator.

$$
\begin{gathered}
k_{15}^{2}=\frac{\pi}{2} \frac{f_{\mathrm{r}}}{f_{\mathrm{a}}} \tan \left(\frac{\pi}{2} \frac{f_{\mathrm{a}}-f_{\mathrm{r}}}{f_{\mathrm{a}}}\right) \\
c_{44}^{\mathrm{D}}=\rho\left(2 t f_{\mathrm{a}}\right)^{2} \\
c_{44}^{\mathrm{E}}=c_{44}^{\mathrm{D}}\left(1-k_{15}^{2}\right)
\end{gathered}
$$

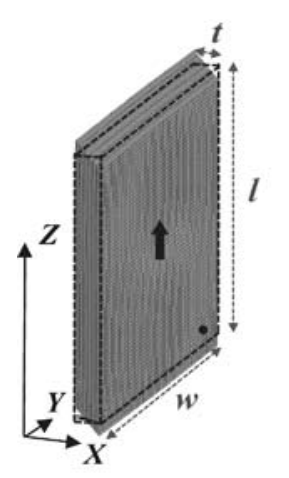

Fig. 3. TS-mode resonator. 


$$
\begin{gathered}
c_{44}^{\mathrm{D}}=\frac{1}{c_{44}^{\mathrm{E}}} \\
\varepsilon_{11}^{\mathrm{T}}=C_{1} \cdot \frac{t}{A} \\
d_{15}=k_{15} \sqrt{s_{44}^{\mathrm{E}} \cdot \varepsilon_{11}^{\mathrm{T}}}
\end{gathered}
$$

\subsection{Length thickness extensional (LTE)-mode resonator}

The LTE model is a long thin strip, as depicted in Fig. 4. Figure 4 also shows the mode shape vibrating along the length or the crystal $Y$ axis. Dimensional parameters should meet the requirements of $w>3 t, l>10 t$ and $l>3 w$. Related material constants are the elastic compliance $s_{11}^{\mathrm{E}}$ and piezoelectric constant $d_{31}$ derived from eqs. (18)-(20). $k_{31}$ is the coupling coefficient of the LTE mode of vibration. . $^{(10,24,27)}$

$$
\begin{gathered}
\frac{k_{31}^{2}-1}{k_{31}^{2}}=\frac{\tan \left(\frac{\pi f_{\mathrm{a}}}{2 f_{\mathrm{r}}}\right)}{\tan \left(\frac{\pi f_{\mathrm{a}}}{2 f_{\mathrm{r}}}\right)} \\
s_{44}^{\mathrm{E}}=\rho\left(2 l f_{\mathrm{r}}\right)^{2} \\
d_{31}=-k_{31} \sqrt{s_{11}^{\mathrm{E}} \cdot \varepsilon_{33}^{\mathrm{T}}}
\end{gathered}
$$

\subsection{XYt45 $5^{\circ}$ and $Z X t 45^{\circ}$ resonators}

To determine the remaining material constants, two more resonator models, $X Y t 45^{\circ}$ and $Z X t 45^{\circ}$, are constructed as illustrated in Fig. 5. They are similar to the LTE resonators but have different crystal axis orientations. The notations for designating the crystal orientation follow the IEEE Piezoelectric Standard defined by the convention that the first two capital letters indicate the principal thickness and the length directions, respectively, of the specimen. ${ }^{(24,26)}$ The third letter $(l, w$, or $t)$ denotes the direction of

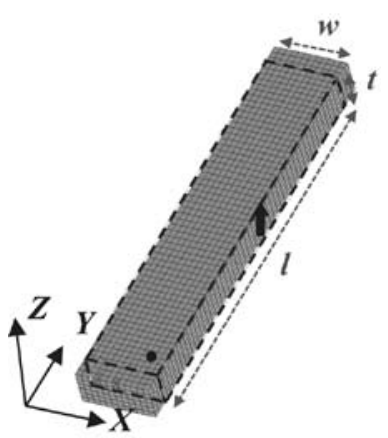

Fig. 4 (left). LTE-mode resonator.

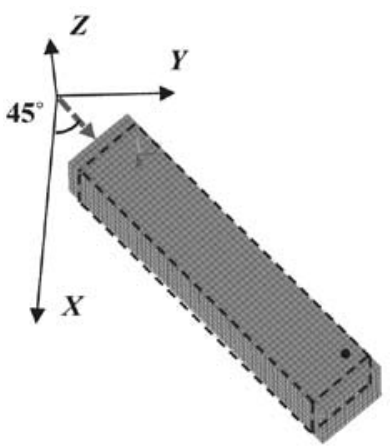

(b)

Fig. 5 (middle and right). (a) $X Y t 45^{\circ}$ resonator and (b) $Z X t 45^{\circ}$ resonator. 
the rotation axis of the strip. The final numerical value is the angle rotated around the rotation axis. For instance, the $X Y t 45^{\circ}$ resonator lies on the crystal $Y Z$ plane with its thickness along the $X$ axis and initial length along the $Y$ axis. Moreover, the strip has been rotated around the thickness direction $t$, i.e., the $X$ axis, by $45^{\circ}$ so that its length is oriented along the direction of the dashed arrow. During the rotation, the polarization direction remains oriented along the $Z$ axis. The mode shapes of these models are similar to the LTE vibration of a strip that undergoes extension and contraction along its length, as presented in Fig. 5. Once the resonant frequencies of these models are identified, the elastic compliance constants $s_{11 X Y 45^{\circ}}^{\mathrm{E}}$ and $s_{11 Z X t 45^{\circ}}^{\mathrm{E}}$ can be derived from eq. (19) for each model. Using the values of $s_{11 X Y t 45^{\circ}}^{\mathrm{E}}$ and $s_{11 Z X 445^{\circ}}^{\mathrm{E}}$ and the material constants derived in previous sections, the remaining elastic compliance constants $s_{13}^{\mathrm{E}}, s_{12}^{\mathrm{E}}$, and $s_{66}^{\mathrm{E}}$ are derived from eqs. (21)-(23). ${ }^{(26)}$

$$
\begin{gathered}
2 s_{13}^{E}=\frac{s_{11 X Y t 45^{\circ}}^{\mathrm{E}}-s_{11}^{\mathrm{E}} \cos ^{4} 45^{\circ}-s_{33}^{\mathrm{E}} \sin ^{4} 45^{\circ}}{\sin ^{2} 45^{\circ} \cos ^{2} 45^{\circ}}-s_{44}^{\mathrm{E}} \\
s_{12}^{\mathrm{E}}=-s_{11}^{\mathrm{E}}+2 \cdot \frac{\left(s_{12}^{\mathrm{E}}\right)^{2}}{s_{33}^{\mathrm{E}}-\frac{1}{c_{33}^{\mathrm{E}}}} \\
s_{66}^{\mathrm{E}}=\frac{s_{11 Z X t 45^{\circ}}^{\mathrm{E}}-s_{11}^{\mathrm{E}}\left(\cos ^{4} 45^{\circ}+\sin 45^{\circ}\right)}{\sin ^{2} 45^{\circ} \cos ^{2} 45^{\circ}}-2 s_{12}^{\mathrm{E}}
\end{gathered}
$$

Through these processes with the six resonator models, all the material constants of PMN-28\%PT single crystals can be derived: six elastic compliance constants $s_{11}^{\mathrm{E}}, s_{12}^{\mathrm{E}}, s_{13}^{\mathrm{E}}$, $s_{33}^{\mathrm{E}}, s_{44}^{\mathrm{E}}, s_{44}^{\mathrm{E}}$, and $s_{66}^{\mathrm{E}}$, three piezoelectric strain constants $d_{31}, d_{33}$, and $d_{15}$, and two dielectric permittivities at constant stress $\varepsilon_{11}^{\mathrm{T}}$, and $\varepsilon_{33}^{\mathrm{T}}$. Then the elastic stiffness matrix at a constant electric field, piezoelectric stress constant matrix, and dielectric permittivity matrix at constant strain, given by eqs. (1)-(3), can be obtained from the relationships in eqs. (24) -(26). In eq. (26), [ ] ${ }^{\text {Tr }}$ denotes a transposed matrix.

$$
\begin{gathered}
{\left[c^{\mathrm{E}}\right]=\left[s^{\mathrm{E}}\right]^{-1}} \\
{[e]=[d]\left[c^{\mathrm{E}}\right]} \\
{\left[\varepsilon^{\mathrm{S}}\right]=\left[\varepsilon^{\mathrm{T}}\right]-[e][d]^{\mathrm{Tr}}}
\end{gathered}
$$

\section{Experimental Resonator Measurement}

Resonator specimens were manufactured following the dimensional requirements described in $\S 3$. PMN-28\%PT single crystals were supplied by iBULe Photonics Co., Ltd. (Incheon, Korea). Figure 6 shows photographs and detailed dimensions of the PMN-28\%PT resonators. The crystals have a density of $8,084 \mathrm{~kg} / \mathrm{m}^{3}$. Five sets of resonators having identical geometries were prepared to ensure the repeatability and reliability of measurements. Moreover, each specimen set was measured five times to confirm the repeatability. All measurements were carried out at room temperature. 


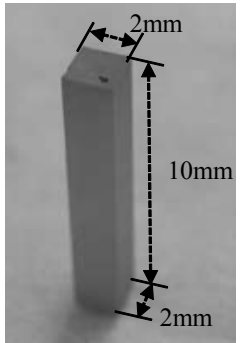

(a)

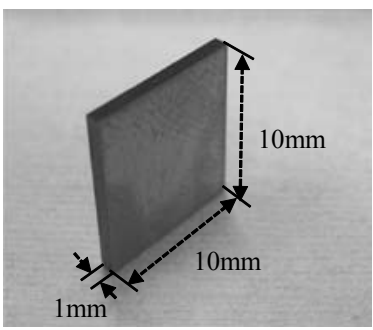

(d)

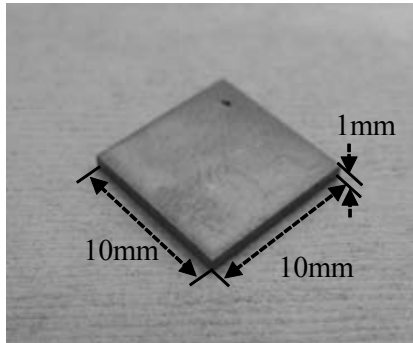

(b)

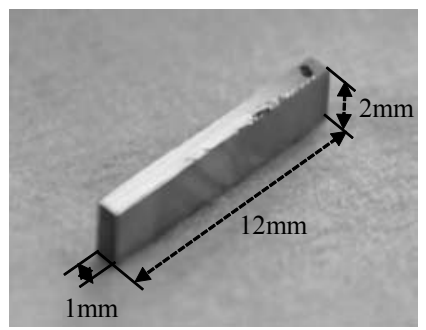

(e)

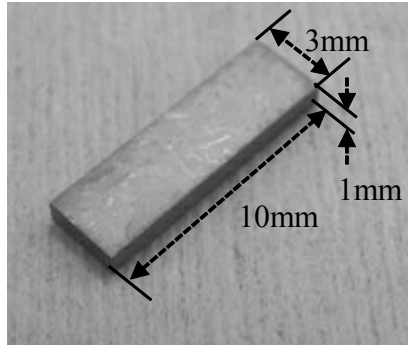

(c)

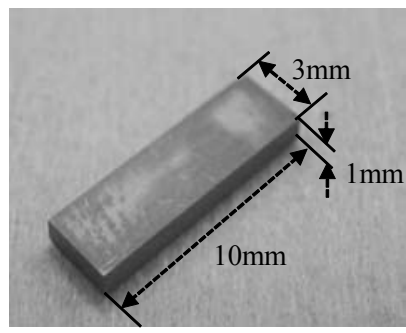

(f)

Fig. 6. Photographs of the six PMN-28\%PT crystal resonators: (a) LE resonator, (b) TE resonator, (c) LTE resonator, (d) TS resonator, (e) $X Y t 45^{\circ}$ resonator, and (f) $Z X t 45^{\circ}$ resonator.

The electrostatic capacitances $C_{3}$ and $C_{1}$ of the crystals were measured with the TE resonator and TS resonator, respectively. Measurements were performed with a 4294A impedance analyzer (Agilent Technologies Inc., Santa Clara, CA). $C_{3}$ was measured to be $4.28 \mathrm{nF}$ and $C_{1}$ was measured to be $1.61 \mathrm{nF} . \varepsilon_{33}^{\mathrm{T}} / \varepsilon_{0}$ was found to be 4841 from eq. (4), and $\varepsilon_{11}^{\mathrm{T}} / \varepsilon_{0}$ to be 1821 from eq. (16).

The impedance spectra of the six resonators were measured for the five sets of specimens. Figures 7-12 show the results, which confirm the repeatability of the measurements. As a representative sample, the spectrum of the TE resonator in Fig. 8 was found to have the mechanical quality factor of 149 . However, a small discrepancy in the measured spectra was observed, particularly in the $X Y t 45^{\circ}$ and $Z X t 45^{\circ}$ resonators, which was attributed to the tolerance in machining the crystal specimens. In order to reduce these experimental errors and thus to increase the accuracy of the determined material constants, the maximum and minimum values of the five measurements were removed, and the remaining three values were averaged to determine the resonant and antiresonant frequencies of each resonator. These truncated mean values of the resonant and antiresonant frequencies, and the discrepancy among the specimen sets were expected to be more robust to experimental errors. The resultant resonant and antiresonant frequencies are summarized in Table 1 for all the resonators. For the $X Y t 45^{\circ}$ and $Z X t 45^{\circ}$ resonators, the antiresonant frequencies are not required to determine the material constants of the PMN-28\%PT crystals.

Once the resonant and antiresonant frequencies are measured, as shown in Table 1, the electromechanical coupling coefficients can be easily calculated. As the most important value of the coupling coefficient, $k_{33}$ of the LE mode has been calculated to be 0.90 from eq. (8) and the data in Table 1. 


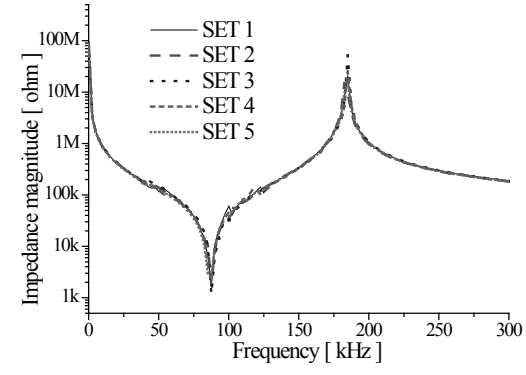

(a)

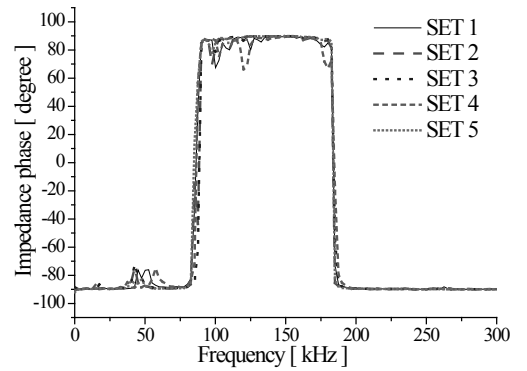

(b)

Fig. 7. Measured impedance spectra of the LE resonators: (a) impedance magnitude and (b) impedance phase.

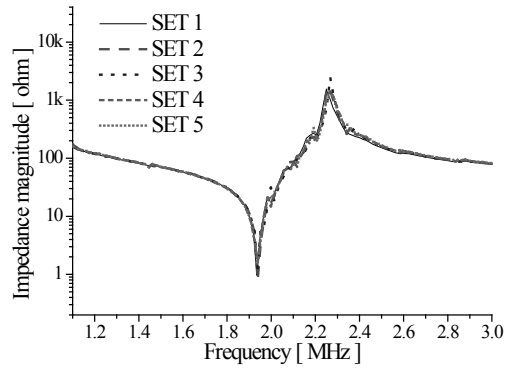

(a)

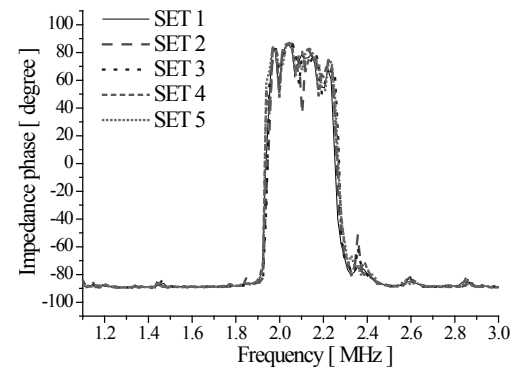

(b)

Fig. 8. Measured impedance spectra of the TE resonators: (a) impedance magnitude and (b) impedance phase.

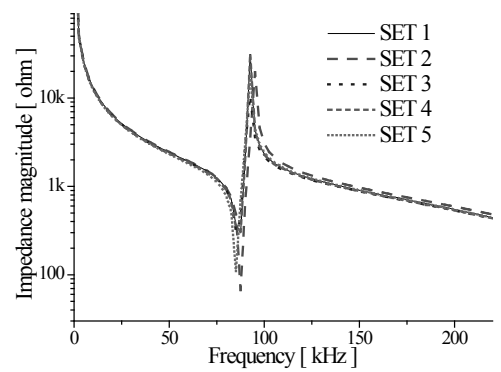

(a)

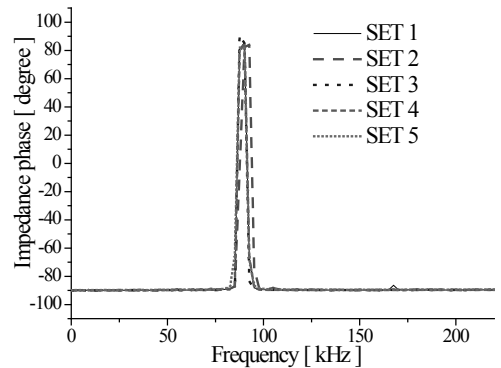

(b)

Fig. 9. Measured impedance spectra of the LTE resonators: (a) impedance magnitude and (b) impedance phase.

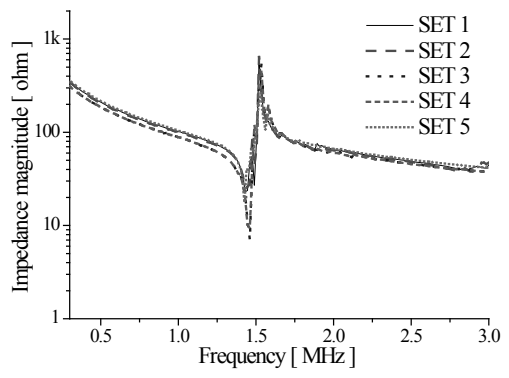

(a)

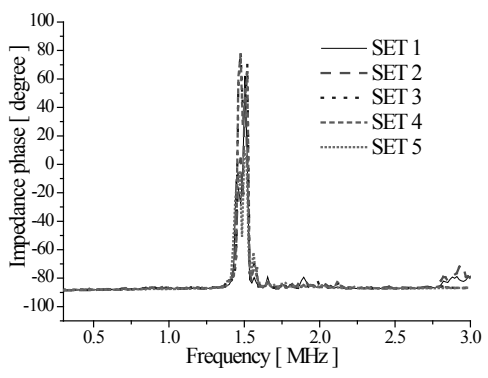

(b)

Fig. 10. Measured impedance spectra of the TS resonators: (a) impedance magnitude and (b) impedance phase. 


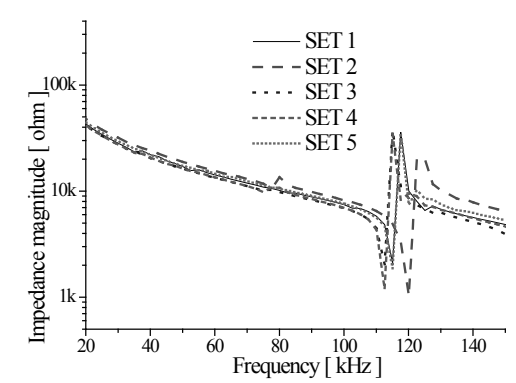

(a)

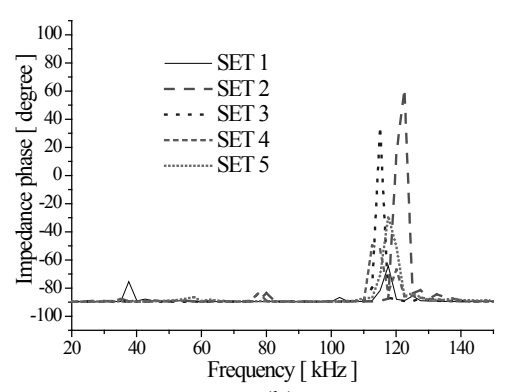

(b)

Fig. 11. Measured impedance spectra of the $X Y t 45^{\circ}$ resonators: (a) impedance magnitude and (b) impedance phase.

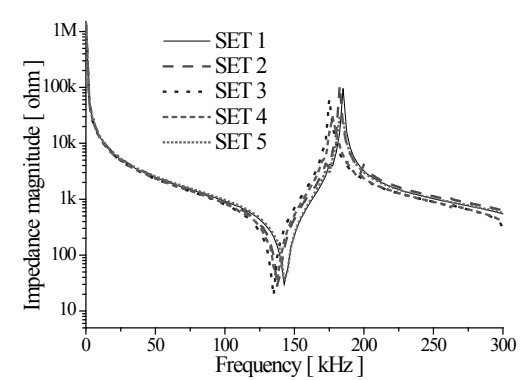

(a)

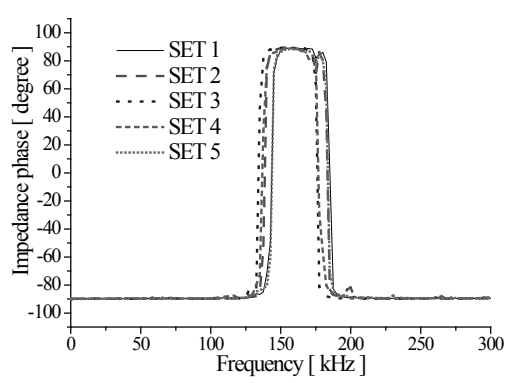

(b)

Fig. 12. Measured impedance spectra of the $Z X t 45^{\circ}$ resonators: (a) impedance magnitude and (b) impedance phase.

Table 1

Resonant and antiresonant frequencies of the six resonators.

\begin{tabular}{lcc}
\hline Resonator type & Resonant frequency $(\mathrm{kHz})$ & Antiresonant frequency $(\mathrm{kHz})$ \\
\hline TE model & 1938 & 2264 \\
LE model & 87.4 & 184.9 \\
TS model & 1449 & 1527 \\
LTE model & 86.4 & 93.1 \\
$X Y t 45^{\circ}$ model & 114.9 & - \\
$Z X t 45^{\circ}$ model & 139.2 & - \\
\hline
\end{tabular}

\section{Determination of Material Constants}

The material constants of the PMN-28\%PT crystals were derived by the methods described in $\S 3$. First, the elastic compliance matrix $\left[s^{\mathrm{E}}\right]$, piezoelectric strain constant matrix $[d]$, and dielectric permittivity matrix at constant stress $\left[\varepsilon^{\mathrm{T}}\right]$ were determined. Then, the elastic stiffness matrix $\left[c^{\mathrm{E}}\right]$, piezoelectric stress constant matrix $[e]$, and dielectric permittivity matrix at constant strain $\left[\varepsilon^{\mathrm{S}}\right]$ were derived from eqs. (24)-(26). The results are summarized in Table 2 . 
Table 2

Material constants of the PMN-28\%PT crystals determined from the resonance measurements.

\begin{tabular}{cccccc}
\hline & $s_{11}^{\mathrm{E}}$ & 41.4 & & $c_{11}^{\mathrm{E}}$ & 14.7 \\
Elastic & $s_{12}^{\mathrm{E}}$ & -22 & Elastic & $c_{12}^{\mathrm{E}}$ & 13.1 \\
compliance & $s_{13}^{\mathrm{E}}$ & -19.4 & stiffness & $c_{13}^{\mathrm{E}}$ & 11.4 \\
constants & $s_{33}^{\mathrm{E}}$ & 47.5 & constants & $c_{33}^{\mathrm{E}}$ & 11.4 \\
{$\left[10^{-12} \mathrm{~m}^{2} / \mathrm{N}\right]$} & $s_{44}^{\mathrm{E}}$ & 15.1 & & $c_{44}^{\mathrm{E}}$ & 6.41 \\
& $s_{66}^{\mathrm{E}}$ & 24.9 & & $\left.c_{66}^{\mathrm{E}} / \mathrm{m}^{2}\right]$ & 4.02 \\
\hline Piezoelectric & $d_{31}$ & -549 & Piezoelectric & $e_{21}$ & -6.88 \\
strain constants & $d_{33}$ & 1282 & stress constants & $e_{33}$ & 21.4 \\
{$\left[10^{-12} \mathrm{C} / \mathrm{N}\right]$} & $d_{15}$ & 169 & {$\left[\mathrm{C} / \mathrm{m}^{2}\right]$} & $e_{15}$ & 11.3 \\
\hline Dielectric & $\varepsilon_{11}^{\mathrm{T}} / \varepsilon_{0}$ & 1821 & Dielectric & $\varepsilon_{11}^{\mathrm{S}} / \varepsilon_{0}$ & 1605 \\
constants & $\varepsilon_{33}^{\mathrm{T}} / \varepsilon_{0}$ & 4841 & constants & $\varepsilon_{33}^{\mathrm{S}} / \varepsilon_{0}$ & 885 \\
\hline
\end{tabular}

\section{Validation through Finite Element Analysis}

The accuracy of the measured data was investigated through finite element analysis (FEA) of the electromechanical impedance of the resonators used in the experiments. Using the commercial software ANSYS ${ }^{\circledR}$, finite element models of the six resonator types were constructed, and harmonic analysis was carried out to calculate the impedance spectra of all the resonator models. The constants in Table 2 were used as material data for the calculations. If the material constants are inaccurate, large discrepancies should be observed between the measured and numerically calculated impedance spectra. It has been reported in many works that the accuracy of the properties of a material can be verified in this way. ${ }^{(13,17,25)}$

Figures 13-18 show the measured and calculated impedance spectra of all six resonators. Among the five sets of resonator specimens used in the measurement, the spectra of the first resonator set (set 3) were used for comparison. The figures show excellent agreement between the two sets of spectra, which confirms the accuracy of the measured material constants. The TE resonator shows the greatest discrepancy, but the difference is only $0.7 \%$ for the resonant frequency and $2.1 \%$ for the antiresonant frequency. The discrepancy is considered to be due to mainly the fluctuation in the measured impedance spectra, which is again considered to be due to the tolerance in machining the crystal specimens.

Hence, overall, the derived material constants can be considered sufficiently accurate to provide the impedance spectra with a maximum error of $2.1 \%$. These comparisons confirm the validity of the resonance measurement technique and the accuracy of the derived material constants in Table 2. 


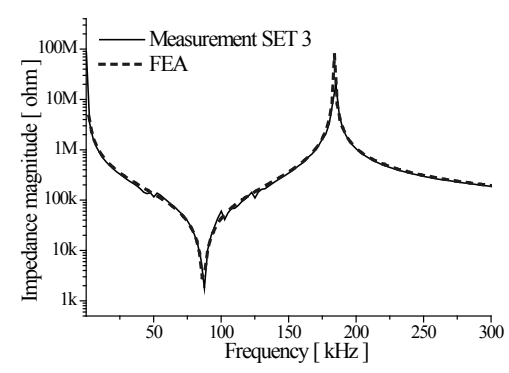

(a)

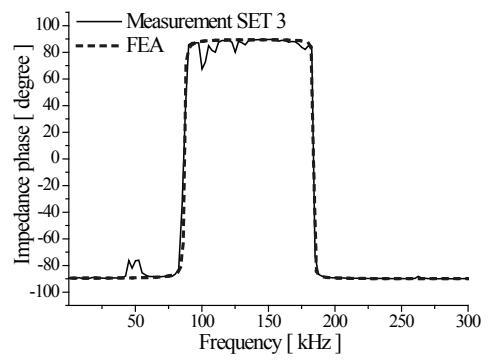

(b)

Fig. 13. Measured and calculated impedance spectra of the LE resonator: (a) impedance magnitude and (b) impedance phase.

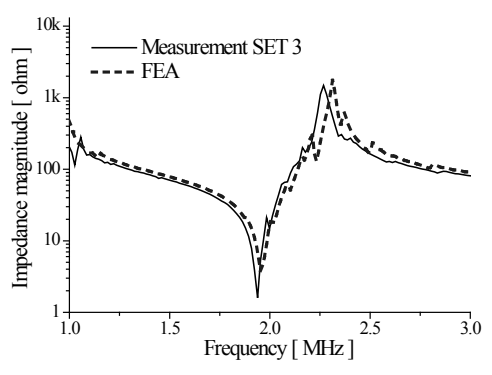

(a)

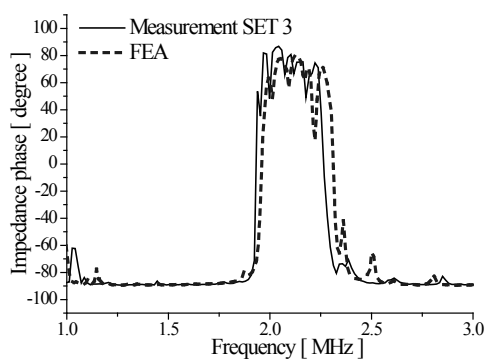

(b)

Fig. 14. Measured and calculated impedance spectra of the TE resonator: (a) impedance magnitude and (b) impedance phase.

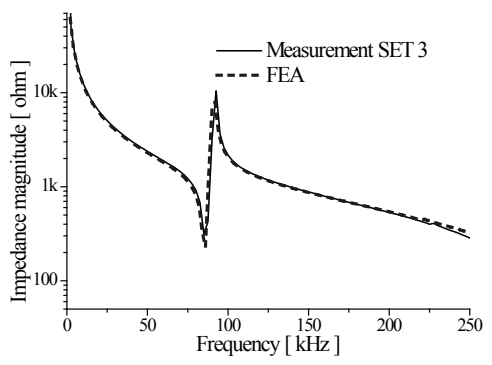

(a)

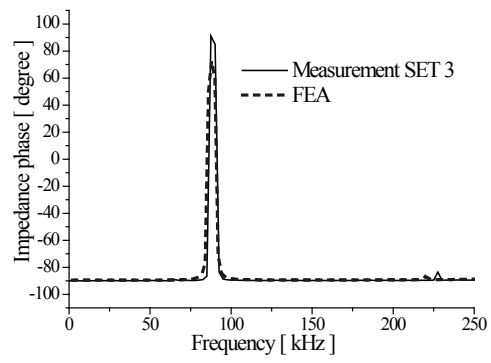

(b)

Fig. 15. Measured and calculated impedance spectra of the LTE resonator: (a) impedance magnitude and (b) impedance phase.

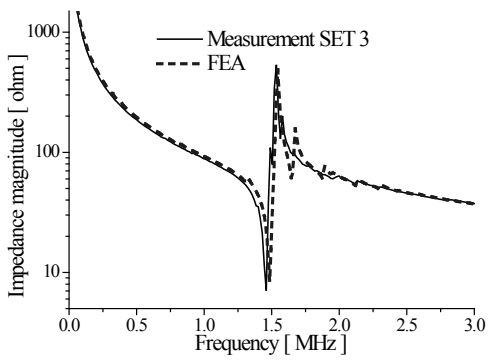

(a)

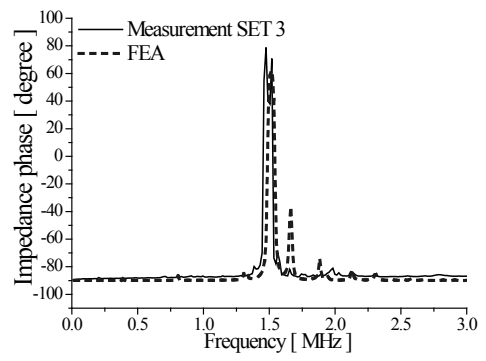

(b)

Fig. 16. Measured and calculated impedance spectra of the TS resonator: (a) impedance magnitude and (b) impedance phase. 


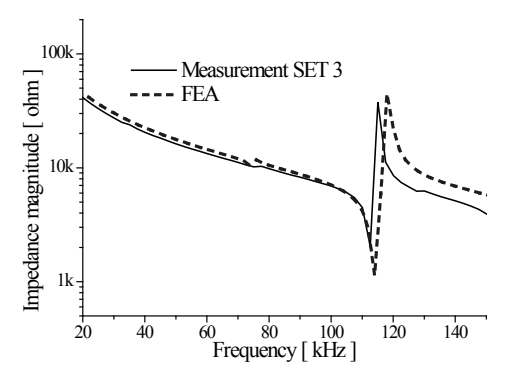

(a)

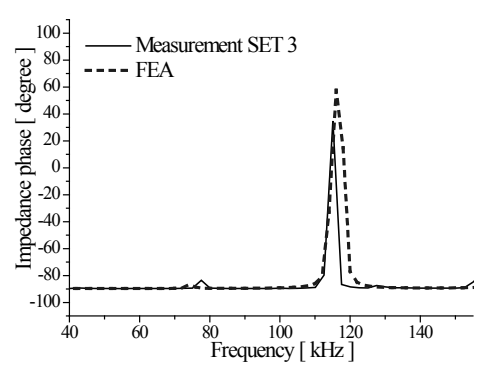

(b)

Fig. 17. Measured and calculated impedance spectra of the $X Y t 45^{\circ}$ resonator: (a) impedance magnitude and (b) impedance phase.

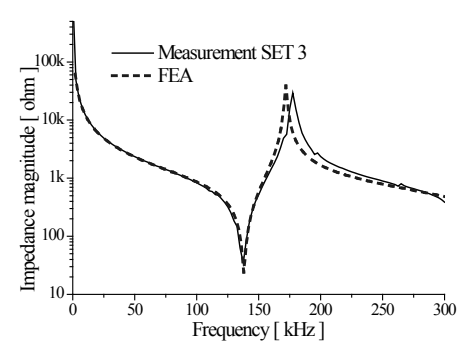

(a)

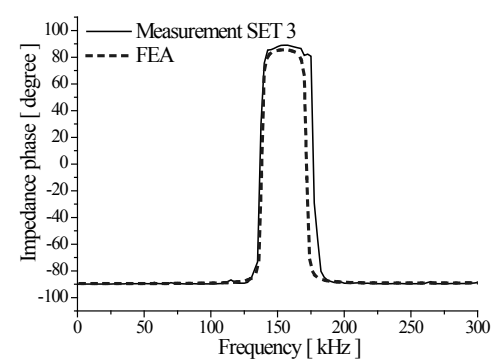

(b)

Fig. 18. Measured and calculated impedance spectra of the $Z X t 45^{\circ}$ resonator: (a) impedance magnitude and (b) impedance phase.

\section{Conclusion}

In this work, all the elastic, piezoelectric, and dielectric constants of PMN-28\%PT single crystals were measured by the resonance method. PMN-28\%PT single crystals were grown by the Bridgman method to have the tetragonal $4 \mathrm{~mm}$ crystal symmetry and thus have eleven independent material constants: six elastic constants, two dielectric constants, and three piezoelectric constants. The eleven constants were determined through measurement and analysis of only the impedance spectra of six different types of PMN-28\%PT resonators in sequence. The method developed in this work could extract all the material constants of PMN-PT crystals through consistent and systematic resonance measurements. To check the validity of the method, experimental impedance spectra of the crystal resonators were compared with the impedance spectra numerically calculated with the derived material constants. The good agreement between the two sets of impedance spectra confirmed the validity of the method and the accuracy of the material constants as well. The material constants determined in this work can improve the accuracy of models used to describe acoustic transducers and facilitate the analysis and design of transducers utilizing PMN-28\%PT crystals. 


\section{Acknowledgements}

This work was supported by the Agency for Defense Development in Korea under contract UD130007DD. The authors appreciate the cooperation of Dr. Sanggoo Lee at iBULe Photonics Co., Ltd., in supplying of the PMN-28\%PT crystal samples.

\section{References}

1 S. E. Park and T. R. Shrout: J. Appl. Phys. 82 (1997) 1804.

2 L. M. Ewart, E. A. McLaughlin, H. C. Robinson, J. J. Stace and A. Amin: IEEE Trans. Ultrason. Ferroelectr. Freq. Control 54 (2007) 2469.

3 M. B. Moffett, H. C. Robinson, J. M. Powers and P. D. Baird: J. Acoust. Soc. Am. 121 (2007) 2591.

4 S. E. Park and T. R. Shrout: IEEE Trans. Ultrason. Ferroelectr. Freq. Control 44 (1997) 1140.

5 W. Jiang, R. Zhang, B. Jiang and W. Cao: Ultrasonics 41 (2003) 55.

6 J. D. Maynard: J. Acoust. Soc. Am. 91 (1992) 1754.

7 X. Jiang, F. Tang, J. T. Wang and T. P. Chen: Physica C 364 (2001) 678.

8 R. Zhang, B. Jiang and W. Cao: J. Appl. Phys. 90 (2001) 3471.

9 X. Geng, T. A. Ritter and S. E. Park: Proc. IEEE Ultrason. Symp., 1998, p. 571.

10 B. Jaffe, W. Cook and H. Jaffe: Piezoelectric Ceramics (Academic Press, New York, 1971) Chap. 3, p. 281.

11 C. Alemany, L. Pardo, B. Jiménez, F. Carmona, J. Mendiola and A. M. González: J. Phys. D: Appl. Phys. 27 (1994) 148.

12 M. Maeda, H. Hashimoto and I. Suzuki: J. Phys. D: Appl. Phys. 36 (2003) 176.

13 T. Lahmer, M. Kaltenbacher, B. Kaltenbacher, R. Lerch and E. Leder: IEEE Trans. Ultrason. Ferroelectr. Freq. Control 55 (2008) 465.

14 K. W. Kwok, H. L. W. Chan and C. L. Choy: IEEE Trans. Ultrason. Ferroelectr. Freq. Control 44 (1997) 733.

15 V. Matko: Sensors 11 (2011) 4474.

16 V. Matko and K. Jezernik: Sensors 12 (2012) 3105.

17 S. Lee and Y. Roh: Jpn. J. Appl. Phys. 46 (2007) 4462.

18 S. L. Swartz and T. R. Shrout: Mater. Res. Bull. 17 (1982) 1245.

19 J. Peng, H. Luo, T. He, H. Xu and D. Lin: Mater. Lett. 59 (2005) 640.

20 M. Shanthi, L. C. Lim, K. K. Rajan and J. Jin: Appl. Phys. Lett. 92 (2008) 142906.

21 S. G. Lee, H. S. Lee, R. G. Monteiro, M. Lee and R. S. Feigelson: AACG/West 16th Conf. Crystal Growth and Epitaxy, 1998.

22 S. G. Lee, R. G. Monteiro, R. S. Feigelson, H. S. Lee, M. Lee and S. E. Park: Appl. Phys. Lett. 74 (1999) 1030.

23 J. F. Nye: Physical Properties of Crystals (Clarendon Press, Oxford, 1985) p. 298.

24 IEEE Standard on Piezoelectricity ANSI/IEEE Std. 176-1978 New IEEE (1978).

25 D. J. Powell, G. L. Wojcik, C. S. Desilets, T. R. Gururaja, K. Guggenberger, S. Sherrit and B. K. Mukherjee: Proc. 1997 IEEE Ultrason. Symp., 1997, p. 1669.

26 T. Ikeda: Fundamentals of Piezoelectricity (Oxford University Press, Oxford, 1996).

27 O. B. Wilson: Introduction to Theory and Design of Sonar Transducers (Peninsular Publishing, Los Altos, 1988) Chap. 5. 